\title{
ELABORAÇÃO DE UM AMBIENTE DIGITAL DE APRENDIZAGEM NA EDUCAÇÃO PROFISSIONALIZANTE EM ENFERMAGEM ${ }^{1}$
}

\author{
DEVELOPING A DIGITAL LEARNING ENVIRONMENT IN NURSING \\ PROFESSIONAL EDUCATION
}

\section{ELABORACIÓN DE UN ENTORNO DIGITAL DE APRENDIZAJE EN LA EDUCACIÓN PROFESIONAL DE ENFERMERÍA}

\author{
Fernanda dos Santos Nogueira de Góes * \\ Luciana Mara Monti Fonseca ${ }^{* *}$ \\ Rosangela Andrade Aukar de Camargo *** \\ Cristina Yuri Nakata Hara *** \\ Jéssica Deponti GobBi ${ }^{* * * * *}$ \\ Angelita Maria Stabile ${ }^{* * * * *}$
}

\begin{abstract}
RESUMO
O desenvolvimento de ambientes de aprendizagem digitais pode contribuir para que o ensino de enfermagem seja mais participativo, disponibilizando ao professor material colaborativo e aos alunos conteúdos e simulações que poderão ser utilizados de acordo com as suas necessidades e ritmos de aprendizagem. Objetivo: Descrever o processo de desenvolvimento de um ambiente de aprendizagem como recurso auxiliar para o professor na formação de alunos de educação profissional de nível médio em enfermagem. Método: Para o desenvolvimento do ambiente de aprendizagem foi utilizado modelo que apresenta quatro fases sequenciais de desenvolvimento de software: definição do escopo, planejamento, produção e implementação, o que facilita didaticamente a construção. Resultados: A definição do escopo foi realizada a partir de resultados de um estudo prévio que identificou as necessidades de aprendizagem dos alunos da educação profissional de nível médio em Ribeirão Preto/SP. Assim optou-se por abordar Sinais Vitais relacionando-os a alguns aspectos da anatomia e fisiologia humana. O sistema multimídia construído para WEB apresenta nível de interatividade plena com simulação construída por meio de cenários e personagem em animação bidimensional (2D). Foi organizado em módulo teórico e um módulo de simulações. Conclusão: Espera-se que o ambiente digital de aprendizagem produzido

\footnotetext{
${ }^{1}$ Projeto de pesquisa vinculado ao Edital CAPEs 024/2010 - Pró-Ensino na Saúde. Titulo: A formação de professores no contexto do SUS: políticas, ações e construção de conhecimento - Pró-Ensino na Saúde.

* Enfermeira, Professora da Escola de Enfermagem de Ribeirão Preto da Universidade de São Paulo. E-mail: fersngoes@ eerp.usp.br

** Enfermeira, Professora da Escola de Enfermagem de Ribeirão Preto da Universidade de São Paulo. E-mail: lumonti@ eerp.usp.br

*** Enfermeira, Professora da Escola de Enfermagem de Ribeirão Preto da Universidade de São Paulo. E-mail: rcamargo@ eerp.usp.br

**** Enfermeira, Maestranda do Programa de Pós Graduação Enfermagem Fundamental da Escola de Enfermagem de Ribeirão Preto da Universidade de São Paulo. E-mail: cristinahara@usp.br

$* * * * *$ Graduanda em Enfermagem da Escola de Enfermagem de Ribeirão Preto da Universidade de São Paulo. E-mail: jessi_gobbi@hotmail.com

****** Enfermeira, Professora da Escola de Enfermagem de Ribeirão Preto da Universidade de São Paulo. E-mail: angelita@ eerp.usp.br
} 
possa agregar conhecimento ao aluno da educação profissional de nível médio em enfermagem bem como ser utilizado pelo professor como recurso pedagógico, facilitando a compreensão de conteúdos abstratos.

Palavras chave: Enfermagem, educação em enfermagem, educação profissionalizante, instrução por computador.

\begin{abstract}
The development of digital learning environments can contribute to nursing education more participative, providing the teacher and students collaborative materials, content and simulations that can be used according to their needs and learning rhythm. Objective: To describe the process of developing a learning environment as a resource to assist the teacher in training professional education students mid-level nursing. Method: For the development of the learning environment was used model that features four sequential phases of software development: scope definition, planning, production and implementation, which facilitates the construction didactically. Results: The definition of the scope was performed based on the results of a previous study that identified the learning needs of students of vocational education average level in Ribeirão Preto/SP. So we chose to address Vital Signs relating them to some aspects of human anatomy and physiology. The multimedia system features built for WEB full interactivity level simulation with built through scenarios and character in twodimensional animation (2D). It was organized in theoretical module and a simulation module. Conclusion: It is expected that the digital environment produced learning can add knowledge to the student of vocational education average level in nursing as well as being used by the teacher as a teaching resource, facilitating the understanding of abstract content.
\end{abstract}

Key words: Nursing, nursing education, education, professional, computer-assisted instruction.

\title{
RESUMEN
}

El desarrollo de entornos digitales de aprendizaje puede contribuir a que la enseñanza de enfermería sea más participativa, proporcionando al profesor materiales de colaboración y a los estudiantes contenidos y simulaciones que pueden usar de acuerdo a sus necesidades y ritmo de aprendizaje. Objetivo: Describir el proceso de desarrollo de un ambiente de aprendizaje como un recurso para ayudar al profesor en la formación de los estudiantes de educación profesional de enfermería de nivel medio. Material y método: Para el desarrollo del entorno de aprendizaje se utilizó el modelo que cuenta con cuatro fases secuenciales de desarrollo de software: definición del alcance, planificación, producción y ejecución, lo que facilita la construcción didácticamente. Resultados: La definición del ámbito de aplicación se realizó en base a los resultados de un estudio anterior que identificó las necesidades de aprendizaje de los estudiantes de nivel medio de formación profesional en Ribeirão Preto/SP. Así se escogió abordar signos vitales relacionados a algunos aspectos de la anatomía y fisiología humana. Las funciones del sistema multimedia construidos para WEB presentan un nivel de interactividad total con una simulación construida a través de escenarios y personajes en la animación de dos dimensiones (2D). Fue organizado en módulo teórico y un módulo de simulación. Conclusión: Se espera que el entorno digital de aprendizaje producido pueda agregar conocimiento a los estudiantes de nivel medio de formación profesional en enfermería, además de ser utilizado por el maestro como recurso didáctico, lo que facilita la comprensión de contenidos abstractos.

Palabras clave: Enfermería, educación en enfermería, educación profesional, instrucción por computador.

Fecha recepción: 05/11/13 Fecha aceptación: 24/02/15 


\section{INTRODUÇÃO}

Dados coletados pelo Conselho Federal de Enfermagem em 2010 demostram que aproximadamente $80 \%$ dos profissionais de enfermagem que atuam no país são de nível técnico, denotando a representatividade dessa força de trabalho na saúde (1).

Faz-se necessário que a formação de nível técnico no Brasil seja permeada pelos princípios norteadores do Sistema Único de Saúde (SUS), pela Lei de Diretrizes e Bases da Educação Profissional e pelas Diretrizes Curriculares Nacionais para a Educação Profissional de Nível Técnico na busca da formação de profissionais éticos, críticos, reflexivos e comprometidos com o sistema de saúde e seus usuários.

Assim, como estratégia de ensino com vistas à adequada qualificação profissional, a inovação tecnológica pode ser um dispositivo que contribua para a apreensão de alguns conteúdos pelos alunos dos cursos profissionalizantes em enfermagem. A informática pode constituir recurso colaborativo ao professor quando incorporadas a metodologias de ensino que estimulem as interações do grupo.

Nesse contexto, considera-se que o desenvolvimento de ambientes digitais de aprendizagem pode contribuir para que o ensino da educação profissional em enfermagem seja mais participativo, disponibilizando ao professor material colaborativo e aos alunos conteúdos e simulações que poderão ser utilizados de acordo com as suas necessidades e ritmos de aprendizagem, além de ferramentas de comunicação síncrona e assíncrona que permitam a troca de informações e experiências entre os usuários, na perspectiva da aprendizagem significativa (2).

Para fins desse estudo utilizou-se a teoria da aprendizagem significativa (2) que considera o estudante ativo na construção do seu conhecimento e do processo educacional, por meio do pensamento reflexivo. Assim, os ambientes de aprendizagem são espaços que estimulam a participação ativa do estudante para observar e modificar as informações, bem como usar a sua intencionalidade no ambiente coletivo, e o professor, como mediador, pode propiciar ambientes com situações complexas próximas ao contexto real (3).

Várias experiências têm demonstrado que o uso de tecnologias na educação em enfermagem pode facilitar o aprendizado e pode influenciar o crescimento da profissão, se incluídos nos objetivos de ensino do curso (4).

Uma revisão sistemática da literatura encontrou 28 estudos publicados entre janeiro de 2001 e dezembro de 2012. Foram identificados três temas: 1) E-aprendizagem dos alunos, 2) tecnologia da informação e comunicação e 3) o desenvolvimento pessoal dos educadores para E-learning. Cada um dos estudos analisados abordou uma variedade de questões que apoiaram e impediu E-learning em programas de enfermagem. Dezenove dos 28 estudos revisados recomenda a incorporação de conhecimentos de informática nos currículos de enfermagem, requisito de enfermeiros que trabalham atualmente em todo o mundo (5).

Desta forma, o objetivo desse estudo é relatar o processo de desenvolvimento de um ambiente de aprendizagem como recurso auxiliar para o professor na formação de alunos de educação profissional de nível médio em enfermagem.

\section{MÉTODO}

Trata-se de estudo metodológico (6) que adotou o referencial da Interação Humano Computador (IHC), que relaciona a ciência da computação, artes, design, ergonomia, psicologia, sociologia, semiótica, linguística, e áreas afins. A interação entre humanos e máquinas acontece através da interface do utilizador, formada por software e hardware (7). 
Para o desenvolvimento do estudo utilizamos referencial pedagógico da problematização, na intenção de problematizar o contexto da formação dos alunos de curso técnico em enfermagem a partir de seu contexto de vida e trabalho, numa tentativa de conduzir os usuários (alunos e professores) à reflexão e ação, procurando transformar a realidade (8).

O estudo se apoia também na teoria da aprendizagem significativa, ao considerar que para haver aprendizagem o aluno precisa ter uma disposição para aprender e o conteúdo a ser aprendido tem que ser potencialmente significativo, ou seja, lógico e psicologicamente significativo. $\mathrm{O}$ significado lógico depende do conteúdo, e o psicológico da experiência individual do aluno (2).

Assim, vislumbramos que a educação em enfermagem, quando auxiliada por ambiente de aprendizagem, pode se beneficiar de práticas pedagógicas nas quais o aluno é sujeito ativo de seu aprendizado, o qual pode ser cooperativo, significativo e facilitar o convívio mútuo (9).

Este estudo envolveu a produção tecnológica de um ambiente de aprendizagem a ser utilizado no contexto da atuação docente junto a alunos do curso de educação profissional de nível médio em enfermagem. Para o desenvolvimento tecnológico utilizamos modelo que apresenta quatro fases sequenciais de desenvolvimento de software: definição do escopo, planejamento, produção e implementação, o que facilita didaticamente a construção (10).

A definição do escopo do ambiente digital de aprendizagem foi realizada a partir dos resultados do estudo prévio, técnicas de brainstorming com os participantes, o que permitiu a definição dos conteúdos a serem incluídos, além de utilização de literatura pertinente. Para tanto, fizemos a opção por apresentar o conteúdo em forma de estudo de caso clínico fictício. Em seguida realizamos a revisão, atualização e reordenação contínua até a elaboração do escopo definitivo.

$\mathrm{Na}$ fase do planejamento foi elaborado o protótipo completo do ambiente digital de aprendizagem. A produção foi realizada por equipe de pesquisadores, responsáveis pela avaliação frequente do material produzido, e por equipe técnica composta de programadores de informática e webdesigner. No que se refere aos recursos de informática foi utilizado multimídia interativa, que possibilita a apresentação e a recuperação da informação e permite ao usuário controlar os elementos da informação que serão transmitidos (11, 12). A fase de implantação tem como objetivo disponibilizar o projeto multimídia para utilização e supervisionar seu funcionamento. A equipe de desenvolvimento realizou os testes funcionais. O ambiente de aprendizagem será disponibilizado em um ambiente Moodle vinculado a uma universidade pública. Desta forma será possível a comunicação entre alunos e professores por meio de e-mails e chat na perspectiva de facilitar a colaboração entre os usuários.

Cabe informar que o este estudo referese a segunda etapa de uma pesquisa maior (4 etapas) e seguiu as normas éticas nacionais e internacionais - Certificado de Apresentação para Apreciação Ética (CAAE) 0177.0.153.000-11.

\section{RESULTADOS}

Para definição do conteúdo que foi abordado no ambiente de aprendizagem foi utilizado os resultados de um estudo que identificou as necessidades de aprendizagem de alunos de um curso de educação profissional de nível médio em enfermagem ${ }^{2}$. Assim, contribuindo com a aprendizagem significativa (2) ali-

${ }^{2}$ Oliveira MCS, Góes FSN. Aprendizagem de alunos de curso técnico e suas interfaces no processo de formação do docente em enfermagem [Internet]. Visualização online de Resumos; 22 a 26 outubro 2012; Ribeirão Preto (Brasil): XX Simpósio Internacional de Iniciação Cientifica da Universidade de São Paulo; [citado em 06 nov 2012]. Disponível em: uspdigital.usp.br/siicusp/cdOnlineTrabalhoObter? numeroInscricao Trabalho $=1139 \&$ numeroEdicao $=20$ \&print $=S$ 
cerçada no princípio de autonomia de Paulo Freire, ou seja, em que o discente é capaz de autogerenciar ou autogovernar seu processo de formação, respeitamos a dignidade do sujeito, ao levar em consideração que o indivíduo é o ser que constrói a sua própria história.

\section{O ambiente de aprendizagem}

Considerando os dados de estudo anterior abordamos o tema sinais vitais relacionando-o a alguns aspectos da anatomia e fisiologia humana. Para isso, é apresentado um caso clínico fictício que se ambienta em uma unidade de saúde e representa a situação de um homem idoso com patologias crônicas comuns ao processo de envelhecimento que poderão ser vivenciadas na prática profissional. O caso foi construído com base em experiências profissionais e apoiado em livros específicos da área de enfermagem, na tentativa de facilitar a articulação entre teoria e prática; apresenta dados gerais para que o usuário possa realizar as simulações propostas para verificação dos sinais vitais e relaciona-las a algumas alterações anatômicas decorrentes do histórico do paciente. Ao navegar pelo caso clínico o aluno tem contato com um paciente idoso, com queixa de cefaleia. O aluno é estimulado a realizar a avaliação clínica do idoso e tem contato com informações do paciente relacionadas a verificação de sinais vitais, hipertensão arterial e arteriosclerose.

Figura 1: Tela inicial do objeto digital de aprendizagem "Sinais Vitais e Anatomia”.

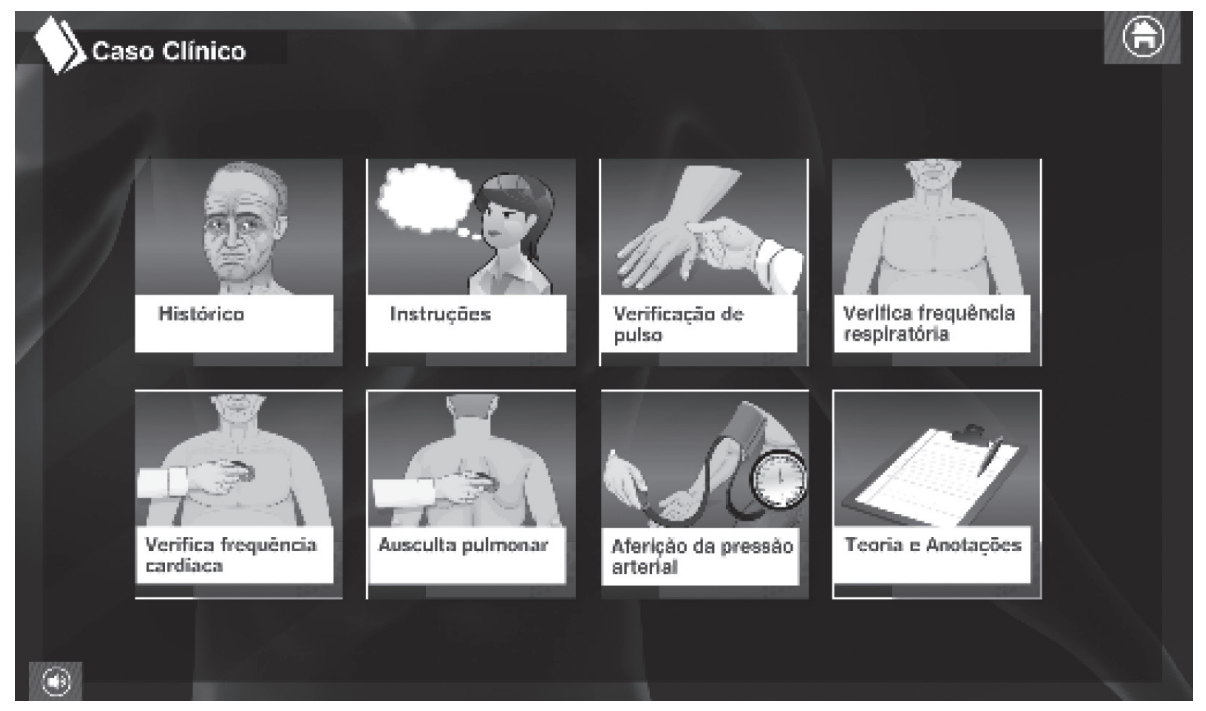

O sistema multimídia construído para WEB apresenta nível de interatividade plena com simulação construída por meio de cenários e personagens em animação bidimensional (2D).

O desenvolvimento do sistema foi realizado por equipe técnica contratada para esse fim, a partir do roteiro e reuniões semanais e envolvimento ativo da equipe de desenvolvi- mento por um período total de desenvolvimento tecnológico de cerca de nove meses. A produção tecnológica foi dividida em três etapas: modelagem 3D e animação, utilizouse o software 3D Studio Max ${ }^{\circledR}$; layout para a criação do layout do game, padrão de cores, botões e telas utilizou-se os softwares Adobe Illustrator ${ }^{\circledR}$, Adobe Edge Animate ${ }^{\circledR}$ e Adobe Photoshop ${ }^{\circledR}$ e programação em HTML5 e 
Javascript, utilizando-se os softwares Adobe Edge Code ${ }^{\circledR}$ e Adobe Dreamweaver ${ }^{\circledR}$.

Foi organizado em módulo teórico e um módulo de simulações. O módulo teórico apresenta a justificativa e os objetivos do ambiente digital de aprendizagem. O módulo de simulação apresenta um jogo educativo digital (cenário de unidade de saúde) com simulações de 5 ações de cuidado relacionadas ao tema, totalizando 7 questões. O usuário, representado por um profissional virtual, observa as situações apresentadas e faz escolha entre as alternativas testando o aprendizado construído. Ao final da avaliação do paciente o usuário tem a oportunidade de realizar um registro de enfermagem. Devido ao formato modular do sistema, o usuário tem a oportunidade de realizar toda a avalição clínica do idoso ou escolher o tema que deseja explorar. As simulações apresentam multimeios, como sons cardíacos e pulmonares, desenhos com visualização interna do coração, pulmão e artéria braquial. Ao final das simulações organizamos uma apresentação sucinta sobre as possíveis alterações anatômicas e funcionais relacionadas ao caso do paciente. Tais ações foram elaboradas como recurso adicional para subsidiar a compreensão do usuário, na tentativa de que ele tenha a possibilidade de relacionar aspectos da anatomia e fisiologia com os sinais e sintomas apresentados pelo paciente, ou seja, articular as questões da teoria e da prática profissional.

$\mathrm{O}$ ambiente digital de aprendizagem apresenta área também para referências, apoio e realização e avaliação do objeto digital.

Figura 2: Tela de simulação de verificação da frequência respiratória do objeto digital de aprendizagem "Sinais Vitais e Anatomia".

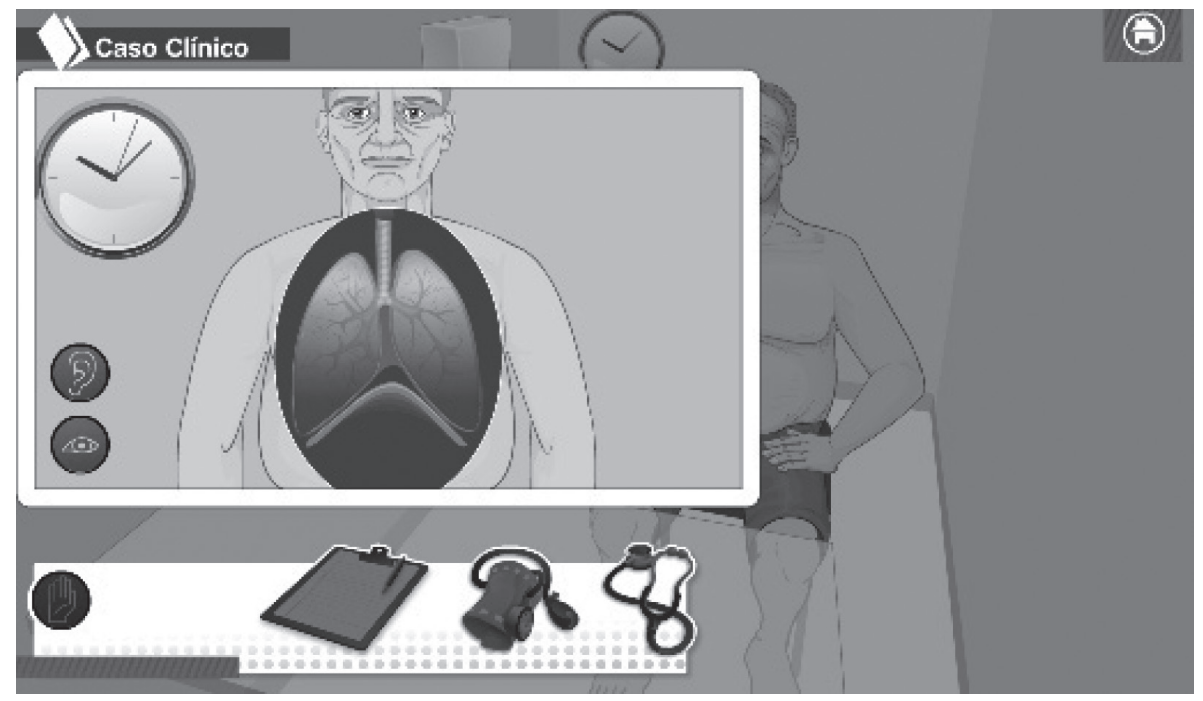

\section{DISCUSSÃO E CONCLUSÕES}

Diante de muitas percepções e relatos na mídia de erros de enfermagem associados ao ensino, acreditamos que se faz necessário repensar as práticas pedagógicas adotadas até o momento e considerar o meio social no qual o ensino de enfermagem, especialmente de nível médio tem se dado. Talvez, os erros recorrentes no cuidado da enfermagem possam ser relacionados à articulação entre políticas econômica e educacional para atender as necessidades de produção para o trabalho 
e desconsideram as questões pedagógicas da formação (13). Além dos motivos citados, há outros que dificultam o exercício da docência na educação profissional em enfermagem de forma que contribua para a aprendizagem significativa dos alunos, ou seja, para que a informação adquirida tenha significado para o usuário por meio de conhecimentos prévios próprios dos alunos (14).

Sabemos que, para que a aprendizagem seja significativa para o aluno, três condições devem ser satisfeitas, a disposição para aprender, conceitos relevantes da cognição do aluno e material educacional que tenha significado lógico e psicológico $(15,16)$.

Nessa perspectiva, pensamos que a construção do ambiente de aprendizagem, enquanto uma estratégia de ensino pode proporcionar a aprendizagem significativa ao representar situações vivenciadas no cotidiano do ensino prático e associa-las aos conteúdos estudados em sala de aula. Adiciona-se também que a elaboração do conteúdo do ambiente de aprendizagem amparado por recursos multimídias avançados e que permitam a interação dos usuários pode facilitar que o estudante compreenda que os conhecimentos construídos são uteis e utilizáveis na sua prática profissional, agregando valor ao trabalho.

$\mathrm{O}$ ambiente de aprendizagem pode ser associado a outros métodos convencionais de ensino e pode proporcionar a aproximação do estudante com situações vivenciadas na prática clínica antes do primeiro contato presencial com o paciente/comunidade (17).

Assim sendo, entre as estratégias de ensino que podem facilitar o processo de ensinar e aprender tem-se a utilização da tecnologia na perspectiva de que estamos vivendo um intenso desenvolvimento tecnológico e, os jovens de hoje tem domínio das linguagens informatizadas, alguns até mesmo antes da linguagem escrita.

Durante a realização desse estudo observamos que a tecnologia tem sido cada vez mais utilizada para a formação e capacitação profissional, especialmente o desenvolvimento de ambiente de aprendizagem o qual se tem intensificado nos últimos anos, principalmente em centros de ensino e pesquisa.

Percebemos que a enfermagem está cada vez mais se inserindo no mundo globalizado e a prática tem se tornado cada vez mais sofisticada e versátil, exigindo flexibilidade dos profissionais (18). A prática profissional sofre diariamente efeitos do desenvolvimento tecnológico e a formação da equipe de enfermagem deve ser capaz de acompanhar essas mudanças.

A opção por construir o ambiente digital a partir dos interesses dos alunos e professores do ensino médio e de graduação em enfermagem tem como base a participação dos sujeitos e a troca de experiencias a partir de situações reais vivenciadas $(2,8-9,13)$. Partimos do pressuposto, que o ambiente digital produzido deveria ter significado para os alunos e professores da educação profissional, haja visto que o desafio do setor educacional é formar cidadãos competentes na aplicação prática de saberes, capacidade de compreender e se enquadrar no mundo e no encontro de soluções para as situações cotidianas sobre as quais se deve refletir para ressignificar o saber (19).

A utilização da tecnologia no processo de ensino-aprendizagem está plenamente justificada se levar em conta que um dos objetivos básicos da educação é preparar os estudantes para serem cidadãos de uma sociedade plural, democrática e tecnologicamente avançada (19). O conhecimento e as tecnologias proliferam atualmente em uma velocidade exponencial, exigindo novas formas de transferência de saberes e uma postura pró-ativa dos docentes e estudantes.

A inserção da tecnologia na educação tem provocado mudanças no aspecto educacional, proporcionando a renovação dos meios de aprendizagem (20). Assim, o uso da tecnologia precisa ser mantido constante dentro da escola. Atualmente, na sociedade, não há mais como separar a tecnologia e sua estreita 
relação com e na educação, estando seu uso em consonância com as novas propostas na educação, pois o ensino tradicional, pouco dinâmico, nem sempre oferece a experiência de trabalho real e o manuseio prático, o que pode ser obtido por meio da instrução por computador.

A utilização de materiais instrucionais pode constituir em hábil instrumento de interação por elucidar aspectos técnicos de doenças e terapêuticas implementadas, oferecendo ao sujeito um modo objetivo de adquirir conhecimentos $(20,21)$. Na seleção da escolha pedagógica para a construção do ambiente de aprendizagem fizemos a opção pela problematização com o propósito maior de preparar o estudante para tomar a consciência do seu mundo e atuar intencionalmente para transformá-lo $(8,22)$. A tecnologia que elaboramos pode facilitar que o aluno aprenda a aprender, e reconstrua sua formação a partir do cotidiano, pois permite que o aluno simule situações comuns, a partir de recursos multimídias, vivenciadas nas atividades práticas e também na atuação profissional.

Admitindo o valor de material educativo na assistência e educação em saúde, pode-se também utilizar o computador como recurso adicional ao professor na formação de profissionais de enfermagem. Em busca de êxito no processo educativo, torna-se imprescindível a utilização de material educativo adequado além de recursos humanos capacitados. Cada vez mais, a tecnologia tem sido incorporada nos ambientes de formação e tem apresentado resultados positivos ao processo ensino-aprendizagem $(9,17,20$, 23).

A educação em enfermagem deve buscar formas de inovar o ensino e que permitam ao estudante se aproximar das situações reais que serão vivenciadas no campo prático o que pode ser obtido por meio de tecnologias digitais $(17,23,24)$. Os avanços tecnológicos induzem a motivação para o desenvolvimento e introdução de novas tecnologias na educação em saúde e no ensino, como os ambientes de aprendizagem. Dessa forma, a tecnologia desenvolvida se constituiu em um sistema multimídia para WEB com nível de interatividade plena no formato de objeto de aprendizagem, com utilização de simulação construída através de cenários e personagem em animação bidimensional (2D).

A construção das simulações do ambiente digital foi desenvolvida a partir de extensivas discussões e reproduziu um modelo do mundo real obtido por meio de fotos de unidades de saúde, que são cenários de práticas de ensino, permitindo um maior realismo. As fotografias, diagramas e desenhos muitas vezes ajudam a esclarecer os conceitos apresentados e devem ser colocadas junto ao texto que se referem (12).

A interatividade que o ambiente digital proporciona revela a necessidade de observação, de concepção e de avaliação dos modos de comunicação que ocorre entre um grupo de pessoas, reforçando a cooperação $(9,17$, 20, 23, 24). A simulação é uma estratégia que visa o aprimoramento da aprendizagem com treinamentos que se aproximam da realidade, onde o aluno coloca em prática o que aprendeu e se torna responsável pela tomada de decisões, humanizando assim o ensino e ajudando o aluno a superar suas dificuldades na prática, o que contribui para a redução de erros nos procedimentos em situações clínicas verídicas (25).

A simulação permite que seus usuários repitam o procedimento quantas vezes for necessário para o domínio da técnica, em um ambiente que permite erros sem arriscar a saúde do paciente aumentando a segurança e o aprimoramento de suas habilidades clínicas. (25). Optamos trabalhar com a simulação computacional, pois acreditamos que essa contribui no preparo de um profissional responsável e de qualidade, com segurança na execução dos procedimentos e significativa melhora no seu desempenho profissional, onde coloca em prática o que aprendeu em sua experiência virtual.

A informatização pode facilitar o preparo 
do estudante para a realidade que irá encontrar no campo de prática, quando conhecimento e habilidades são necessários para lidar com situações análogas à realidade (9, 25).

A qualidade dos cuidados exige conhecimentos e competências dos profissionais de saúde que possibilitem a intervenção segura, contribuindo para minimizar o erro (25). Como cenário de prática do ambiente digital de aprendizagem decidimos trabalhar com uma unidade de saúde, já que é o local que mais se aproxima das experiências da vida real do aluno, sendo que para sua formação todos devem passar por estágios em uma unidade de saúde. Contudo, o ambiente digital torna-se um método eficiente de treinamento que permite um pensamento reflexivo junto com interações sociais sem julgamentos, que permite ao estudante perceber como e onde ocorreram erros que poderiam ter sido evitados e reconhecer que competências devem ser desenvolvidas.

A utilização de ambiente de aprendizagens deve permitir que o usuário estabeleça seu ritmo de aprendizagem, ou seja, ele deve ter o direito de escolher seu local de estudo, estabelecer seu ritmo de aprendizagem e ter acesso a informações coerentes e interação $(9,26)$. Assim, acreditamos que ambiente de aprendizagem apresentado nesse estudo atenderá a tais critérios, pois será disponibilizado na Internet e poderá ser utilizado como aplicativo. Além disso, o fato do ambiente digital não ser construído de forma linear permite ao usuário interagir da forma como julgar adequada com liberdade de escolha.

O conteúdo do ambiente digital foi elaborado a partir das expressões dos usuários finais o que pode colaborar com a aprendizagem significativa. Espera-se que o material produzido possa agregar conhecimento ao aluno da educação profissional de nível médio em enfermagem bem como ser utilizado pelo professor como recurso pedagógico, facilitando a compreensão de conteúdos abstratos.
Esperamos que esta estratégia de ensino contribua para a formação de um profissional crítico-reflexivo e que seja capaz de executar suas ações a partir da significação do seu aprendizado mediado pela informática. A utilização da tecnologia enquanto uma ferramenta de ensino auxiliar ao professor pode estimular a prática reflexiva, possibilitando que o aluno execute as atividades práticas com segurança e conscientes das implicações éticas e sociais.

Acreditamos na importância desse ambiente de aprendizagem enquanto ferramenta de apoio ao ensino na área da enfermagem, pois oferece ambiente interativo e diferenciado com abordagem que propicia a articulação teoria e prática.

\section{REFERÊNCIAS}

1. Conselho Federal de Enfermagem. Análise de dados dos profissionais de enfermagem existentes nos Conselhos Regionais [Internet]. Brasilia; 2011 [citado 01 abril 2013]. Disponivel em: http://site.portalcofen.gov.br/sites/default/files/pesquisaprofissionais.pdf.

2. Ausubel DP. A aprendizagem significativa: a teoria de David Ausubel. São Paulo, SP: Moraes; 2006.

3. Jonassen DH. Computadores, ferramentas cognitivas: desenvolver o pensamento crítico nas escolas. Porto, PT: Porto Ed.; 2007.

4. Frota NM, Barros LM, Araújo TM, Caldini LN, Nascimento JC, Caetano JF. Construction of an educational technology for teaching about nursing on peripheral venepuncture. Rev Gaucha Enferm. 2013; 34(2): 29-36.

5. Button D, Harrington A, Belan I. E-learning \& information communication technology (ICT) in nursing education: a review of the literature. Nurse Educ Today. 2014; 34(10): 1311-23. 
6. LoBiondo-Wood G, Haber J. Nursing Research. Methods, critical appraisal, and utilization. 8th ed. St Louis: Mosby; 2013.

7. Preece J, Rogers Y, Sharp H. Design de Interação: além da interação homemcomputador. Porto Alegre/RS: Bookman; 2013.

8. Freire P. Educação como prática da liberdade. 30a ed. Rio de Janeiro/RS: Paz e Terra; 2007.

9. Cogo ALP, Pedro ENF, Silva APSS, Specht AM, Valli GP. Tecnologias digitais no ensino de graduação em enfermagem: as possibilidades metodológicas por docentes. Rev. Eletr. Enf. 2011; 13(4): 657-64.

10. Bernardo V. Metodologia para desenvolvimento de projeto multimídia aplicado ao ensino da medicina. [Dissertação de Mestrado]. [São Paulo/SP]: Universidade Federal de São Paulo; 1996.

11. Fava R. Educação 3.0: como ensinar estudantes com culturas tão diferentes. São Paulo: Editora Tanta Tinta; 2012.

12. Ribeiro N. Multimédia e Tecnologias Interativas. $5^{\mathrm{a}}$ ed. Lisboa: FCA - Editora Informática; 2012. $664 \mathrm{p}$.

13. Wermelinger G, Amancio Filho A, Machado $\mathrm{MH}$, Oliveira E. Transformações do mundo do trabalho e a formação em saúde. Divulgação em Saúde para Debate. 2012; 47: 92-101.

14. Miranda KCL, Barroso MGT. A contribuição de Paulo Freire à prática e educação crítica em enfermagem. Rev Lat Am Enfermagem. 2004; 12(4): 631-35.

15. Gariglio JA, Burnier S. Saberes da docência na educação profissional e tecnológica: um estudo sobre o olhar dos professores. Educ. rev. 2012; 28(1).

16. Nobrega-Therrien SM, Guerreiro MGS, Moreira TMM, Almeida MI. Projeto Político Pedagógico: concepção, construção e avaliação na enfermagem. Rev Esc Enferm USP. 2010; 44(3): 679-86.
17. Fonseca LMM, Del'Angelo N, Góes, FSN, Ferreira FSC, Martins CPDG.R, Silvan SCG. Avaliação clínica do prematuro: opinião dos estudantes de enfermagem acerca de um software educacional. Cienc enferm. 2012; XVIII (2): 83-91.

18. Paravic KT. Enfermería y Globalización. Cienc enferm. 2010; XVI (1): 9-15.

19. Trevizan MA, Mendes IAC, Mazzo A, Ventura CAA. Investment in nursing human assets: education and minds of the future. Rev Lat Am Enfermagem. 2010; 18(3): 467-71.

20. Silva NCM, Denise Hollanda I, Resck SMR, Soares MI, Souza-Junior DI, Vieira NF. Estratégias de ensino das terapias alternativas e complementares na graduação em Enfermagem: revisão integrativa. Rev. Eletr. Enf. 2013; 15(4): 1061-7.

21. Palhares GEM, Haueisen SM, Paolinelli FCR. Educação Permanente em Saúde: Reflexões e desafios. Cienc enferm. 2010; XVI (2): 25-33.

22. Berbel, NAN, Sánchez GSA. A metodologia da problematização com o Arco de Maguerez: uma perspectiva teórica e epistemológica. Filosofia e Educação. 2011; 3: 264-87.

23. Galvão ECF, Puschel VAA. Aplicativo multimedia em plataforma móvel para o ensino da mensuração da pressão venosa central. Rev Esc Enferm USP. 2012; 46(esp): 107-15.

24. Lévy P. Cibercultura. São Paulo: Editora $34 ; 2010$.

25. Martins JCA, Mazzo A, Baptista RCN, Coutinho VRD, Godoy S, Mendes IAM et al. A experiência clínica simulada no ensino de enfermagem: retrospectiva histórica. Acta Paul Enf. 2012; 25(4): 619-25.

26. Blake H. Computer-based learning objects in healthcare: the student experience. Int J Nurs Educ Scholarsh. 2010; 7(1): $1-15$. 